\title{
Measurements of UV-A radiation and hazard limits from some types of outdoor lamps
}

\author{
E. El-Moghazy, A.-E.A. Abd-Elmageed ${ }^{\star}$, and S. Reda \\ Photometry and Radiometry division, National Institute for Standards (NIS), Giza, Egypt
}

Received: 1 February 2014 / Accepted: 15 September 2014

\begin{abstract}
Illumination using artificial light sources is common in these days. Many manufactures are paying for the design of lamps depending on high efficacy and low UV hazards. This research is focusing on the most useable lamps in the Egyptian markets; high pressure mercury (HPM), metal Halide (MH), and high pressure sodium (HPS). A set up for relative spectral power distribution based on single monochromator and UVA silicon detector for absolute irradiance measurements are used. The absolute irradiance in $\left(\mathrm{W} / \mathrm{m}^{2}\right)$ in UVA region of the lamps and their accompanied standard uncertainty are evaluated.
\end{abstract}

Keywords: Artificial sources, illumination, UVA radiation, irradiance, uncertainty

\section{Introduction}

The high intensity discharge (HID) lamps such as high pressure mercury (HPM), high pressure sodium (HPS) and metal Halide (MH) lamps mainly designed for outdoor lighting where we need high efficacy and high luminous flux $[1,2]$.

High pressure mercury lamps (see Fig. 1) have a short quartz glass discharge tube that contains a mixture of argon and mercury. The discharge produces ultraviolet radiation specially the near ultraviolet. As a light source, they are relatively compact, which allows their light to be controlled via optical equipment $[1,3]$.

On the other hand, high pressure sodium lamps (see Fig. 2) were developed and introduced as energy efficient sources in road lighting, industrial lighting, and security application. Nowadays, HPS lamps are also appropriate for many interior applications, particularly where color rendering is not essential concern due to their high efficiency and long life. In the lamp design, the exterior bulb protects the arc tube from changes and drafts in temperature, prevents oxidation of the internal parts. This makes HPS lamps especially easy to use in many fixture types $[1,3]$.

Metal halide lamps (see Fig. 3) are a further development of mercury lamps. Apart from mercury, they also contain a mixture of metal halides to improve luminous efficacy and to enhance color rendering. They have excellent luminous efficacy and good color rendering qualities; their nominal lamp life is high. They are extremely compact light sources, whose light can be easily controlled $[1,3,4]$.

^Correspondence: alaa_nis@yahoo.com

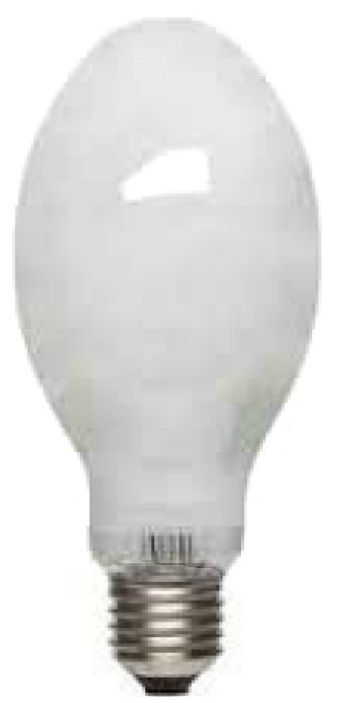

Fig. 1. High pressure mercury lamp.

This research present the study of three types of HID lamps from OSRAM in terms of their irradiance and spectral power distribution for ultraviolet radiation present in their emitted radiation. Lamps HPM 125 Watt, HPS 150 Watt and MH 150 Watt are used as they are safe use in the outdoor lighting [5].

In general, UV light has three wavelength regions UVA (315 nm-400 nm), UVB (280 nm-315 nm) and UVC (200 nm-280 nm). Glass stop the UVB and UVC, while transpire portion of UVA $[1,6]$ so we will concern only with the UVA portion. Different parameters are measured such as UVA absolute irradiance and relative spectral power distribution. 


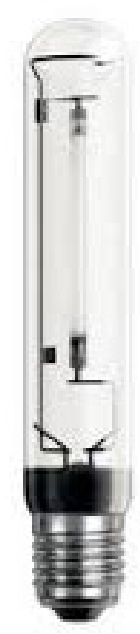

Fig. 2. High pressure sodium lamp.

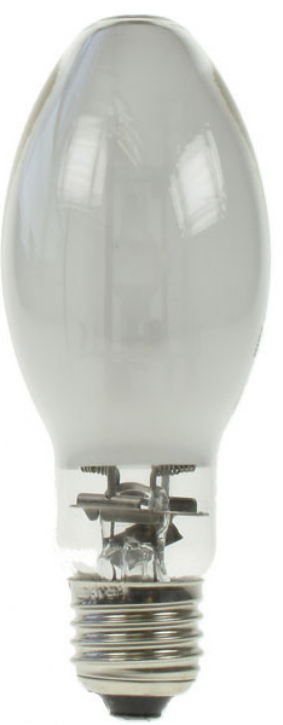

Fig. 3. Metal halide lamp.

Spectral irradiance in UVA region is defined as the power of electromagnetic radiation per unit area in $\left(\mathrm{W} / \mathrm{m}^{2} / \mathrm{nm}\right)$ hence,

$$
\text { UVA irradiance } E_{\lambda}(\lambda)=\int_{\lambda_{1}}^{\lambda_{2}} E(\lambda) d \lambda
$$

where, $E_{\lambda}(\lambda)$ is spectral irradiance in $\left(\mathrm{W} / \mathrm{m}^{2} / \mathrm{nm}\right)$.

On the other hand, spectral power distribution (SPD) measurement describes the power per unit area per unit wavelength of an illumination (radiant exitance). More specifically, the concentration is a function of wavelength to any radiometric quantity or photometric quantity $[2,7]$.

\section{Materials and methods}

The setup and monochromator function illustrated at (Fig. 4) is to measure the radiation from the three spectral

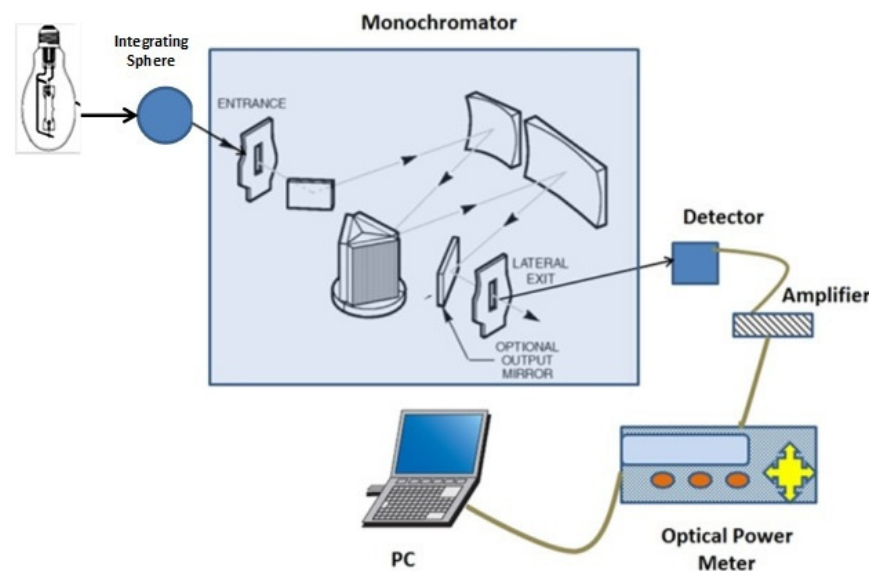

Fig. 4. Schematic diagram of NIS Facility for measuring spectral power distribution.

lamps produces narrow and intense lines. This radiation is introduced into an integrating sphere to build a uniform, monochromatic, and nearly Lambertian source of high spectral radiant flux. The integrating sphere of the entrance optics is needed in order to uniformly irradiate the entrance slit of the monochromator as a function of the irradiance of the source independent from source geometry. In the used spectral range, two 1200 lines/mm gratings (blaze wavelengths $250 \mathrm{~nm}$ and $750 \mathrm{~nm}$ ) were used to analyze the input spectrum of the lamps to their spectral power distribution while the data was collected through the exit slit. At the exit slit of the monochromator, a silicon ( $\mathrm{Si}$ ) detector is used to detect the output signal. The lamps was aged according to IES publication [8] then each lamp aligned at $50 \mathrm{~cm}$ in front of the integrating sphere entrance slit of a system based on MS257 single monochromator from Newport Corporation. The system was adjusted to acquire readings at the range from $200 \mathrm{~nm}$ to $800 \mathrm{~nm}$ with step $2 \mathrm{~nm}$, also this monochromator band pass was adjusted nominally at $4 \mathrm{~nm}$ resulting slit function uncertainty $\pm 0.34 \%$ at $k=2[9]$.

According to CIE [5], UVA irradiance can be calculated spectrally and the total radiant exposure to the eye shall not exceed $10000 \mathrm{~J} / \mathrm{m}^{2}$ for exposure times less than $1000 \mathrm{~s}$.

The equation of the total radiant exposure is:

$$
\begin{gathered}
E_{\mathrm{UVA}} t=\sum_{315}^{400} \sum_{t} E_{\lambda}(\lambda, t) \Delta t \Delta \lambda \leqslant 10000 \mathrm{~J} \mathrm{~m}^{-2} \\
(t<1000 \mathrm{~s})
\end{gathered}
$$

hence, $E_{\mathrm{UVA}} \leq 10 \mathrm{~W} / \mathrm{m}^{2}(t \geq 1000 \mathrm{~s})$, where:

$E_{\lambda}(\lambda, t)$ is the spectral irradiance in $\mathrm{W} / \mathrm{m}^{2} / \mathrm{nm}$;

$\Delta \lambda$ is the bandwidth in $\mathrm{nm}$;

$t$ is the exposure duration in seconds.

Irradiance levels hazard for $E_{\mathrm{UVA}}$ in $\mathrm{W} / \mathrm{m}^{2}$ according to CIE [5] are classified into Exempt $=10$, Low risk $=33$, and Mod risk $=100$. 


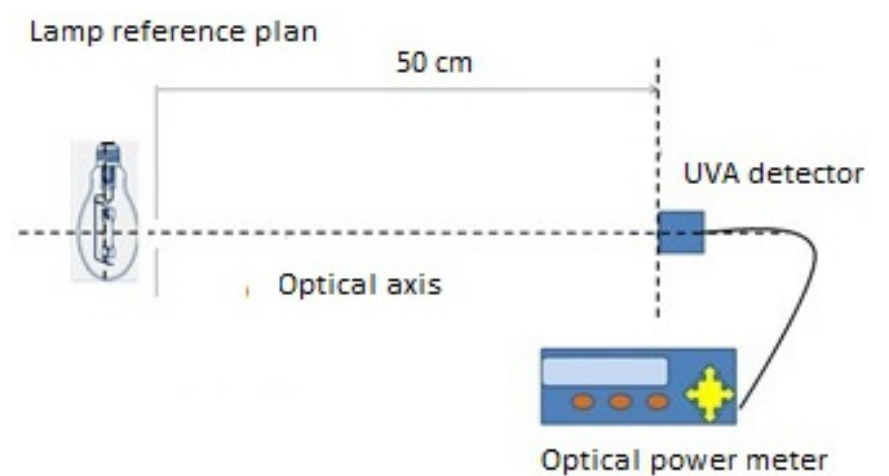

Fig. 5. Schematic diagram of NIS facility for measuring UVA irradiance using calibrated radiometer.

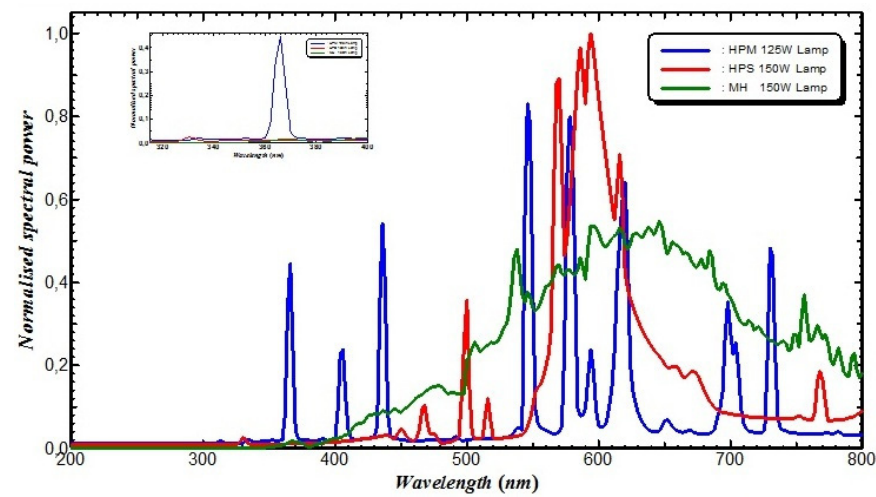

Fig. 6. Normalized spectral power distribution.

The absolute spectral irradiance level of each lamp in the UVA range at $50 \mathrm{~cm}$ is measured using a calibrated radiometer model 268 UVA from UDT Company (see Fig. 5).

\section{Results}

The acquired relative spectral power distribution was normalized for each lamp so we can compare the lamps (see Fig. 6). Results show that HPM emits the largest amount of UVA compared with MH and HPS lamps. While HPS emits the lowest amount with moderate amount was from MH lamp. The amount of UVA irradiance is measured absolutely at distance $50 \mathrm{~cm}$ and the quantities illustrated in (Fig. 7).

Also, according to (Eq. (2)), it is highly recommended to use these lamps only in outdoor, as they may be lie in the range of the CIE hazard condition if used indoor. By using inverse square law, Table 1 shows the calculated value of the minimum distance for the three lamps to be used in the safe limit.

\section{Uncertainty analysis}

The associated uncertainty must be quoted whenever the results of a measurement are reported. This tells the

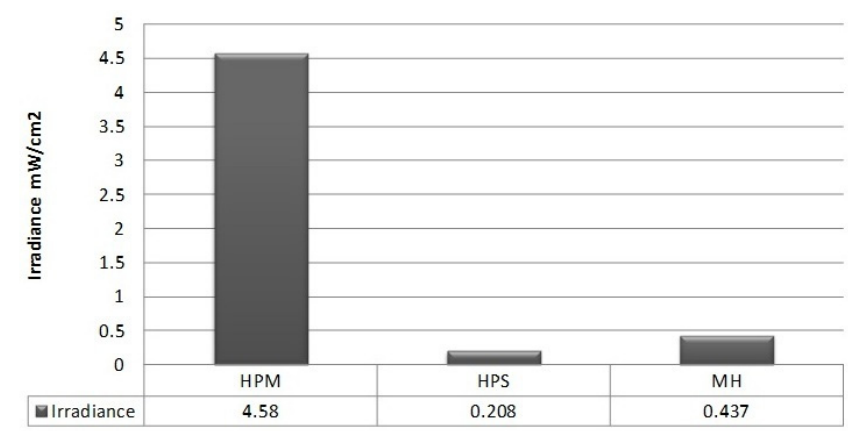

Fig. 7. UVA absolute irradiance levels Using UVA radiometer at $50 \mathrm{~cm}$.

Table 1. Calculated safe limit distance for the three lamps.

\begin{tabular}{cc}
\hline Lamp & Safe limit \\
\hline HPM & $>107 \mathrm{~cm}$ \\
HPS & $>33 \mathrm{~cm}$ \\
$\mathrm{MH}$ & $>23 \mathrm{~cm}$ \\
\hline
\end{tabular}

user of the precision with which the measurement was made. Uncertainty analysis is thus a fundamental part of metrology.

Evaluation of the uncertainty is done by the Guide to the expression of Uncertainty in Measurement (GUM) method. This method is adopted and described in details by International Organization for Standardization (ISO) (ISO, 1993) [10].

The standard uncertainty $u\left(x_{i}\right)$ to be associated with input quantity $x_{i}$ is the estimated standard deviation of the mean $[10,11]$

$$
u\left(x_{i}\right)=s\left(\bar{X}_{i}\right)=\left(\frac{1}{n(n-1)} \sum_{k=1}^{n}\left(X_{i, k}-\bar{X}_{i}\right)^{2}\right)^{1 / 2} .
$$

The combined standard uncertainty $u_{c}(y)$ is obtained by combining the individual standard uncertainties $u_{i}$, these can be evaluatedet as Type A and Type B.

That is,

$$
u_{c}^{2}(y)=\sum_{i=1}^{N}\left(\frac{\partial f}{\partial x_{\mathrm{i}}}\right)^{2} u^{2}\left(x_{i}\right)
$$

Uncertainty model used for the determination of the UVA irradiance $E_{\mathrm{UVA}}(\lambda)$ is:

$$
E_{\mathrm{UVA}}(\lambda)=E_{s}(\lambda)+\delta E_{l}+\delta E_{r}+\delta E_{i}
$$

where,

$E_{s}(\lambda)=$ uncertainty due to reference spectral irradiance UVA standard radiometer (obtained from the calibration certificate).

$\delta E_{l}=$ distance effect on the irradiance measurements (calculated by using the inverse square law).

$\delta E_{r}=$ repatibility of the measurements (standard deviation of repeated 5 times). 
Table 2. UVA irradiance uncertainty budget.

\begin{tabular}{cc}
\hline Uncertainty component & $\begin{array}{c}\text { Relative standard } \\
\text { uncertainty } \%\end{array}$ \\
\hline Irradiance responsivity calibration of standard radiometer $\left(E_{S}\right)$ & 1.24 \\
Current regulation of lamp $\left(\delta E_{i}\right)$ & 0.32 \\
Distance measurements $\left(\delta E_{l}\right)$ & 0.01 \\
Repeatability $\left(\delta E_{r}\right)$ & 0.01 \\
Expanded uncertainty $(k=2)$ & 2.56 \\
\hline
\end{tabular}

$\delta E_{i}=$ effect of current regulation of lamp on the irradiance measurements (division of $V-I$ by $V$-flux slopes [4]).

The uncertainty budget of the absolute irradiance measurements is shown at Table 2 with confidence level $95 \%$ $(k=2)$.

\section{Discussion}

The absolute irradiance measurements of UVA region as well as the relative spectral power distribution of three outdoor lighting lamps from OSRAM are carried out. Due to different content material inside the arc tube for each type of lamps, the relative spectral power distributions of the three lamps are completely different (see Fig. 6). The absolute irradiance measurements at $50 \mathrm{~cm}$ in $\mathrm{W} / \mathrm{m}^{2}$ in UVA region from each lamp are obtained, using UVA standard radiometer with maximum responsivity at $365 \mathrm{~nm}$ (see Fig. 7). It clarify that HPM emits the largest amount of UVA compared with MH and HPS lamps. While HPS emit the lowest amount with moderate amount was from MH lamp. On the other hand, the safety distance required to use these lamps are calculated according to the CIE recommendation of hazard levels in UVA region (see Tab. 1). It is noticed that the safety distance is proportional with the amount of UVA emission. The measurements were performed under control of environmental conditions and good regulation of electrical power. The accompanied uncertainty in the absolute irradiance measurements is $\pm 2.56 \%$ (see Tab. 2).

\section{Conclusion}

Three different types of lamps commonly used in the outdoor lighting from OSRAM are studied to assess their unwanted output in the UVA region. These lamps are designed mainly to emit their power in the visible region. Practically, they emit the majority of their energy in the visible region but part of their energy is emitted in the UVA region. A set up based on single monochromator and UVA standard radiometer for relative spectral power distribution and absolute irradiance measurements are used, respectively. It is found that each of the three lamps has its own characteristics, and they emit most of their spectrums in the visible region with different spectral distributions. Results show that HPM emits the largest amount of UVA compared with MH and HPS lamps. While HPS emit the lowest amount with moderate amount was from MH lamp. The UVA level hazards for each lamp are calculated according to CIE recommendation, and the safety distance was obtained from the measurement of the absolute irradiance emission of UVA. Hence, it is highly recommended to use these lamps only in outdoor, as they may be lie in the range of the CIE hazard condition if used indoor. The minimum safe limit distance according to Table 1 must be taken into consideration.

\section{References}

1. C. De Cusatis, Handbook of Applied Photometry (Optical Society of America, Poughkeepsie, New York, 1994)

2. F. Grum, R. Becherer, Optical Radiation Measurements (Academic Press, Radiometry, 1979), Vol. 1

3. Lighting Associates, Inc, http://www.lightingassociates.org

4. E.E.M. El-moghazy, A technical study of the development of working standard lamps for lighting applications, Doctoral thesis, College of Woman, Ein Shames university, 2012

5. Photobiological safety of lamps and lamp systems, standard CIE S 009/E (2002)

6. P. Sharma, V.K. Jaiswal, H.C. Kandpal, Ultraviolet radiation emitted by compact fluorescence lamps, J. Metrol. Soc. India (MAPAN) 24, 183-191 (2009)

7. A. Abd-Elmageed, Detector-based traceability chain for spectral irradiance using tunable laser based facility at PTB, Doctoral thesis, Braunschweig University, Germany, 2011

8. IES Guide to Lamp Seasoning, J. Illuminating Engineering society, IES LM-54-12 (2012)

9. S.M. Reda, A.-E.A. Abd-Elmageed, On the use of spectral lamp lines in the Monochromator Calibration, Trends in Advanced Science and Engineering, TASE 4, 22-25 (2012)

10. International Organization for Standardization (ISO), Guide to the expression of uncertainty in measurement (1993)

11. United Kingdom Accreditation Service (UKAS), The expression of uncertainty and confidence in measurement, 2nd edn. (2007) 